\title{
Pain on Injection of Propofol: Efficacy of Paracetamol and Lidocaine
}

\author{
H. Khouadja1,2, H. Arnous ${ }^{1}$, K. Tarmiz ${ }^{1}$, D. Beletaifa ${ }^{1}$, A. Brahim ${ }^{1}$, W. Brahem ${ }^{1}$, J. Sakhri², \\ K. Ben Jazia 1 \\ ${ }^{1}$ Department of Anesthesiology and Intensive Care, CHU Farhat Hached, Sousse, Tunisia \\ ${ }^{2}$ Research Unit UR12SP32, CHU Farhat Hached, Sousse, Tunisia \\ Email: hosny kh@yahoo.fr
}

Received 9 December 2013; revised 3 March 2014; accepted 21 March 2014

Copyright (C) 2014 by authors and Scientific Research Publishing Inc.

This work is licensed under the Creative Commons Attribution International License (CC BY).

http://creativecommons.org/licenses/by/4.0/

(c) (i) Open Access

\begin{abstract}
Background and Objectives: Propofol remains the most common drug for induction of general anaesthesia, although it causes considerable pain on injection. None of the commonly used methods completely attenuate this discomfort. We aimed to investigate the effect of i.v. paracetamol pretreatment on the propofol injection pain. Materials and Methods: A prospective randomized double-blind study was conducted on 180 patients, ASA I or II status, scheduled to undergo elective surgery. They were randomly assigned to one of the three groups of 60 each. Groups I, II, III were pretreated with $40 \mathrm{mg}$ of lidocaine in saline, $100 \mathrm{mg}$ of paracetamol and $10 \mathrm{ml}$ of saline, respectively. All patients had an 18-gauge catheter inserted into a superficial radial vein. After 2 min of venous occlusion, one-fourth of the total propofol dose was injected into the vein over a period of 20 seconds. During the injection of both pretreatment solution and propofol, a blinded researcher assessed the patient's pain level using a four-point verbal rating scale (VRS) (none $=0$, mild $=1$, moderate $=2$, and severe $=3$ ). $X^{2}$ test and Kruskal-Wallis tests were used for the statistical analysis. For all analyses, differences were considered to be significant at $P<0.05$. Results: The three groups were comparable in respect to patient's characteristics. The incidence of pain on injection of propofol in placebo, i.v. paracetamol and lidocaïne groups were $85 \%, 36 \%, 21 \%$ respectively $(p<0.05)$. Intensity and severity of propofol induced pain were comparable between paracetamol and lidocaine groups. Conclusion: Pretreatment using i.v. paracetamol was found to be effective in reducing propofol injection-induced pain.
\end{abstract}

\section{Keywords}

Propofol, Injection, Pain, Paracetamol, Lidocaine, Anaesthesia 


\section{Introduction}

Propofol (2, 6-diisopropylphenol) remains a popular choice for induction and maintenance of general anesthesia owing to its rapid onset, short duration, easy titration, and favorable profile for side effects [1]. However, during induction of anesthesia pain is a common problem that can be very distressing to the patient. Its incidence has been reported to range from $28 \%$ to $90 \%$ [2]. The physiopathology of this pain is attributed till this moment to one and a combination of more than one of 3 proposed mechanisms. The first mechanism relates pain to the triggering of the local Kallikrein-kinin cascade. Another suggested mechanism was the stimulation of the nociceptive receptors at the free nerve endings located between the intima and the media layers of the venous wall. The third proposed mechanism relates pain to the $\mathrm{pH}$ and concentration of propofol [3].

As a result, several interventions have been investigated to alleviate the pain associated with propofol injection. These include the addition of lidocaine to propofol, cooling or warming the drug, pretreatment with ephedrine, ondansetron, metoclopramide, opioids, thiopental, ketamine, ketorolac, dexamethasone [3]-[6]...

Since, pretreatment using intravenous (i.v.) injection of lidocaïne at $0.5 \mathrm{mg} / \mathrm{kg}$ in conjunction with venous occlusion is suggested to be as the most effective intervention [7]-[9].

Paracetamol (N-acetyl-p-amino-phenol) is widely used for pain management. Although the exact mechanism of action of paracetamol is not yet clear, its clinical effects most likely arise from central action. Recently, it has been demonstrated a weak peripheral effects by blocking impulse generation within the bradykinin-sensitive chemoreceptors responsible for the generation of nociceptive impulses [10].

These proposed mechanisms prompted us to investigate the effect of i.v. paracetamol on propofol injection pain. We, therefore, aimed to compare the effect of i.v. paracetamol with that of lidocaïne for the prevention of propofol-induced pain during induction of anesthesia.

\section{Methods}

After approval of the local Ethics Committee and have obtained the informed, oral consent and in writing, of the patient, we conducted a prospective randomized double-blinded study between July 2011 and November 2012 at Farhat Hached teaching hospital. We included 180 patients, ASA I or II status, scheduled for general anesthesia for elective surgery. Patients were allocated to one of the three groups of 60 each. None of the patients was premedicated before entering the operation room. After routine monitoring, an 18-gauge catheter was inserted into a superficial radial vein and a pneumatic tourniquet (pressure inflated to $70 \mathrm{mmHg}$ ) placed on the upper arm, to occlude the venous drainage. The patients were pretreated over a period of 10 seconds (sec.) with one of the pretreatment solutions: $10 \mathrm{ml}$ of normal saline (Group I), $40 \mathrm{mg}$ of lidocaïne diluted to $10 \mathrm{ml}$ (Group II), or 100 $\mathrm{mg}(10 \mathrm{ml})$ of paracetamol (Group III). After $2 \mathrm{~min}$, the occlusion was released and one-fourth of the total calculated dose of propofol (Propofol lipuro ${ }^{\circledR} 1 \%$ B'Braun) was delivered through the i.v. line over a period of 20 sec. During the injection of pretreatment or propofol, a clinician blinded to the group assignment, evaluated pain using a four- point verbal rating scale (VRS) (none $=0$, mild $=1$, moderate $=2$, and severe $=3$ ) and noticing any behavioral signs associated. Behavioral signs were considered as VRS $=3$, when the patient had tears, arm withdrawal, strong vocal response or responses accompanied with facial grimacing. Thereafter induction of anesthesia was continued.

Within $24 \mathrm{~h}$ after the operation, the injection site was checked for pain, edema, or allergic reaction by an anesthesiologist who was blinded to the group assignment.

The primary outcome variable was the incidence of moderate to severe pain reported. Based on previous reports, we suggested that paracetamol will reduce the incidence of propofol induced pain by 50 percent when compared to saline. A sample size of 180 achieves $80 \%$ power to detect a significant difference among groups at "alpha” of 0.05 using the two-degree freedom $\mathrm{X}^{2}$ test. Clinical characteristics among the groups were compared using one-way ANOVA or the Kruskal-Wallis test. The incidence of moderate/severe pain and the number of patients reporting following propofol injection, the incidence of behavioral signs, and other adverse effects were compared using a Chi Square analysis. $\mathrm{P}<0.05$ was considered significant.

\section{Results}

Of 180 patients screened for eligibility, no one was excluded from the study. There was no significant difference in demographic data between the groups (Table 1). Overall results for incidence and intensity of pain during injection of pretreatment solution in the three groups are shown in Figure 1, Figure 2. There was no difference in the 
Table 1. Demographic data.

\begin{tabular}{ccccc}
\hline Groups & Placebo & Lidocaine & Paracetamol & $\boldsymbol{p}$ \\
\hline Age & $43.6 \pm 15.1$ & $42.8 \pm 13.1$ & $41.6 \pm 12.7$ & 0.129 \\
Height (cm) & $163.2 \pm 8.3$ & $163.1 \pm 6.7$ & $165.9 \pm 8.5$ & 0.147 \\
Weight (kg) & $68.2 \pm 12.7$ & $68.8 \pm 11.7$ & $68.1 \pm 10.8$ & 0.891 \\
ASA & & & \\
I & $38(63.3 \%)$ & $43(71.7 \%)$ & $42(70 \%)$ & 0.583 \\
II & $22(36.7 \%)$ & $17(28.3 \%)$ & $18(30 \%)$ & 0.586 \\
Sex & & & & \\
Male & $32(53.3 \%)$ & $30(50 \%)$ & $29(48.3 \%)$ & 0.5 \\
Female & $28(46.7 \%)$ & $30(50 \%)$ & $31(51.7 \%)$ & 0.667 \\
\hline
\end{tabular}

Data are presented as mean $( \pm \mathrm{SD})$

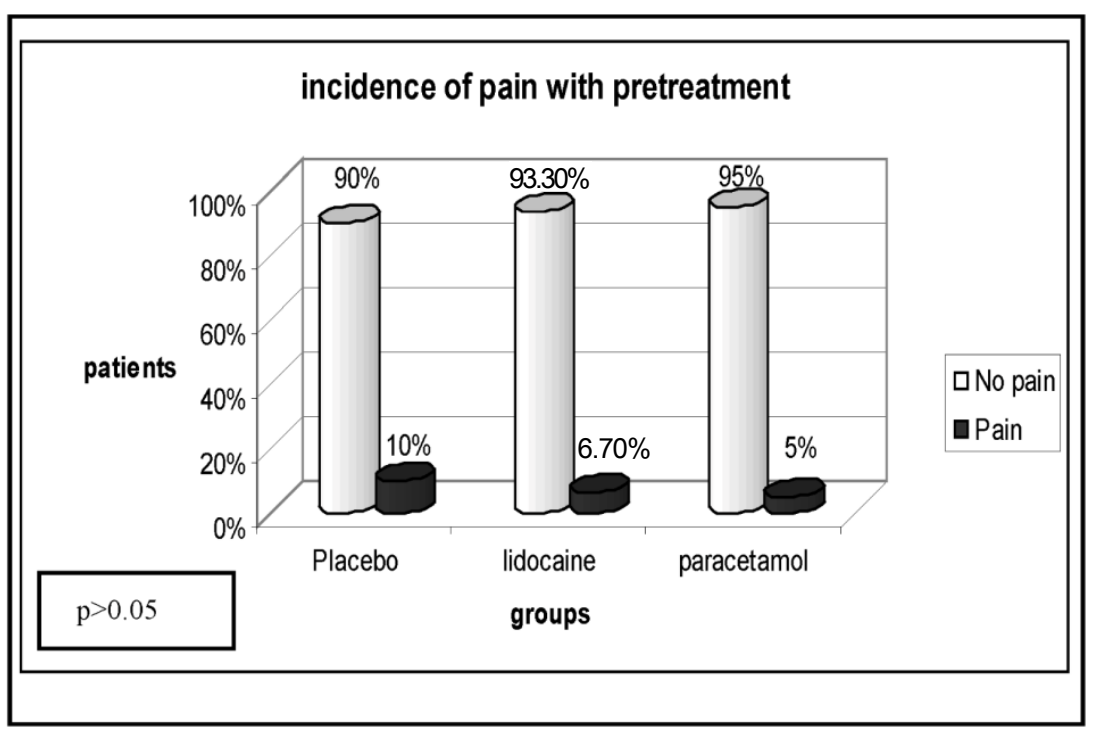

( $p>0.05$ paracetamol vs lidocaine and placebo)

Figure 1. Incidence of pain with pretreatment.

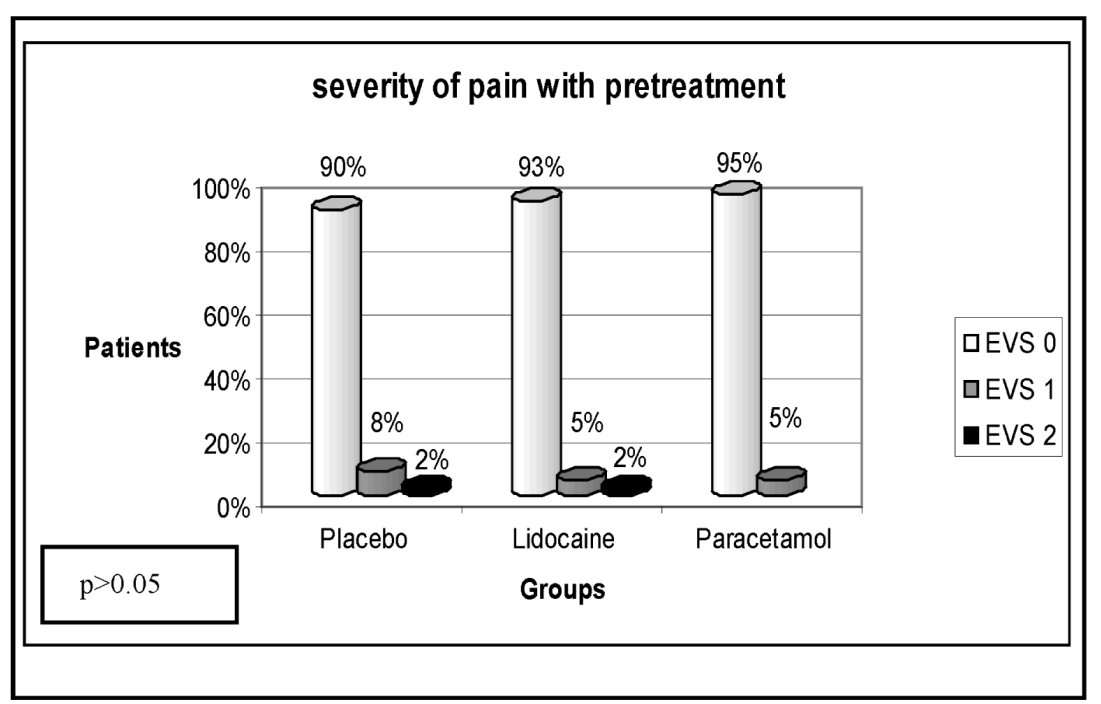

( $>0.05$ paracetamol vs lidocaine and placebo)

Figure 2. Severity of pain with pretreatment. 
incidence of pain between the three groups (10\% in Group I, 6.7\% in Group II and 5\% in Group III (p > 0.05)). Pretreatment with lidocaine produced mild pain in $5 \%$ and moderate pain in $2 \%$ of the patient. Pretreatment with saline solution produced mild pain in $8 \%$ and moderate pain in $2 \%$ of the patient. When we evaluated Pretreatment with paracetamol, intensity of pain was only mild in $5 \%$ of the patient.

The data on the severity and incidence of propofol-induced pain are given in Table 2 . The overall incidence of pain was $85 \%$ in the control group, $21.7 \%$ in the lidocaïne group and $36.6 \%$ in the paracetamol group ( $<$ 0.05). The intensity of pain was severe in $35 \%$ of patients in the control group, $8.3 \%$ in the paracetamol group and $1.7 \%$ in the lidocaine group $(\mathrm{p}<0.01)$.

When we compared propofol-induced pain in Group II and III, severity and intensity of pain were similar ( $21.7 \%$ and $36.6 \%$ respectively, $\mathrm{p}=0.21 \%$ ) (Figure 3 ).

We did not observe any complications such as pain, edema, rush or allergic reaction at the injection site in the first $24 \mathrm{~h}$ after the operation in the three groups.

\section{Discussion}

In our study, we found that i.v. Paracetamol was effective in decreasing the incidence of pain during i.v. injection of propofol. But, when compared to lidocaine, paracetamol failed to prove any benefit to reduce propofol-induced pain.

This side effect is a major concern to anesthesiologists and patients. While admittedly not a serious complication, pain on injection of propofol has been ranked by American anaesthesiologists as the seventh most important problem of current clinical anaesthesiology [11].

Table 2. Incidence and severity of propofol induced pain.

\begin{tabular}{ccccc}
\hline & Placebo group & Lidocaine group & Paracetamol group & $\boldsymbol{p}$ \\
\hline EVS 0 & $9(15 \%)$ & $47(78.3 \%)$ & $38(63.3 \%)$ & $<0.01$ \\
EVS 1 & $14(23.3 \%)$ & $7(11.7 \%)$ & $11(18.3 \%)$ & $<0.01$ \\
EVS 2 & $16(26.7 \%)$ & $5(8.3 \%)$ & $6(10 \%)$ & $<0.01$ \\
EVS 3 & $21(35 \%)$ & $1(1.7 \%)$ & $5(8.3 \%)$ & $<0.01$ \\
EVS $>$ 0 & $51(85 \%)$ & $13(21.7 \%)$ & $22(36.6 \%)$ & $<0.01$ \\
\hline $\mathbf{1}+\mathbf{2}+\mathbf{3})$ & & & & \\
\hline
\end{tabular}

$(\mathrm{p}<0.05$ drugs vs control)

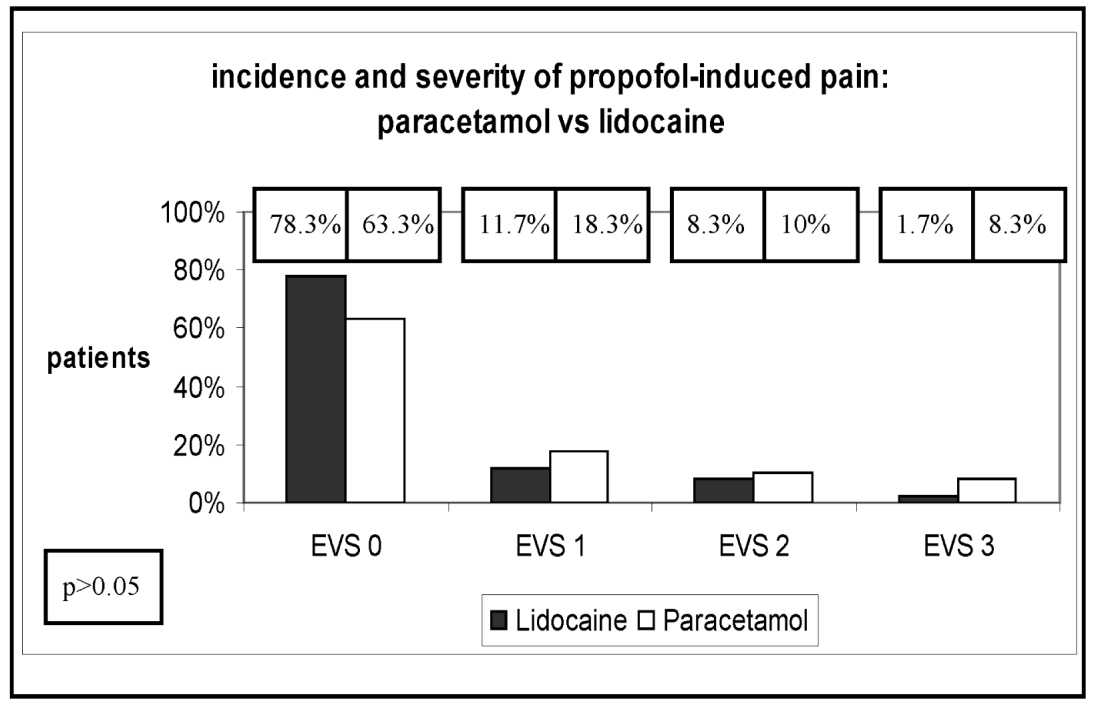

( $>0.05$ IV paracetamol vs lidocaine)

Figure 3. Incidence and severity of propofol induced pain: Paracetamol vs Lidocaine. 
The mechanism by which propofol causes pain on injection is still poorly understood [6]. It is thought to correlate with the amount of propofol in the aqueous phase. The initial component involves immediate stimulation of nociceptors and free nerve endings. The delayed component of pain, appearing within half a minute, is caused by bradykinine generation associated with the activation of the plasma kallikrein-kinin system [12]. Bradykinin produces local vasodilatation and hyperpermeability, which may, therefore, increase the contact between the aqueous phase propofol and free nerve endings, resulting in pain on injection. Ando, et al. have suggested that propofol-induced pain occurred in response to prostanoids, particularly prostaglandin E2 [13].

Intrinsic factors that are associated with pain on injection of lipid emulsion propofol include temperature (either cooling or warming), injection site (e.g. a large vein) and solvent (medium-chain triglyceride/long-chain triglyceride vs. long-chain triglyceride) [3]. Of these factors, warming and medium-chain triglyceride/long-chain triglyceride solvent, as well as lower $\mathrm{pH}$, are known to decrease the free propofol concentration in the aqueous phase, and hence reduce propofol-induced pain [4] [14]-[16]. In our study, we chose to use Propofol-lipuro® which is an emulsion of propofol $1 \%$ in a mixture of long-chain and medium-chain triglycerides (Lipofundin_ MCT 10\% B Braun Ltd., Melsungen, Germany). In addition, the concentration of free propofol in the aqueous phase is reduced when formulated in Lipofundin and may explain the observations that propofol-Lipuro ${ }^{\circledR}$ causes less pain on injection [17] [18].

The nature of pain can be extremely aching, burning and crushing. In our study, severe pain (VRS = 3) was reported by $35 \%$ of patient in the control group.

Various methods have been attempted to attenuate this injection pain. They include prior administration of opioid, lidocaine, prilocaine, thiopental, ketamine, metoclopramide, ondansetron, and ephedrine. These methods do not eradicate pain completely, but do decrease the incidence or severity of injection pain. Of these several agents, lidocaine is the most commonly used in clinical practice [19]. The most common method used in routine is the adding of 10 - $40 \mathrm{mg}$ lidocaine to the syringe of propofol immediately prior to use. In this case, lidocaine decreases the $\mathrm{pH}$ of lipid emulsion propofol, which in turn decreased free propofol concentration in the aqueous phase [20]. However, the maximum licensed dose of lidocaine is $10 \mathrm{mg}$, which is often exceeded, and addition of lidocaine may destabilise the emulsion formulation of propofol with a potential risk of causing pulmonary fat embolism [17]. Lidocaine may also induce cardiovascular, neurotoxic and other complications. However, less than $1 \mathrm{mg} / \mathrm{kg}$ can be used safely [20].

To alleviate propofol injection pain, lidocaine can also be used as a pretreatment. It is presumed that preinjected lidocaine acts mostly as a local anaesthetic. Sasaki, et al. noted that the analgesic effect of preinjected lidocaine increased when a tourniquet was used concomitantly [21]. A meta-analysis conducted by Picard and Tramèr confirmed that lidocaine should be administered with a rubber tourniquet before propofol injection for the most effective pain prevention [22]. Moreover, Kaya, et al. showed that pretreatment with lidocaine plus venous occlusion for 60 seconds significantly reduced the incidence of propofol-induced pain compared with lidocaine without venous occlusion [8].

In our study, we applied $40 \mathrm{mg}$ of lidocaine plus venous occlusion for 120 seconds. Our results showed a beneficial effect of adding lidocaine to microemulsion propofol in reducing pain during injection.

Paracetamol (N-acetyl-p-amino-phenol) is a well tolerated drug with proven efficacy. Despites the popularity of this medicine, the mechanism by which Paracetamol achieves its effects is still debated. It has antipyretic and analgesic effects but lacks anti-inflammatory effects. The mechanism of action of paracetamol analgesia is multifactorial. Though the exact mechanism is not yet clear, its clinical effects most likely arise from central action. The exact site and mode of action within the central nervous system (CNS) is poorly defined and even controversial. It has weak peripheral effects, which have been recently demonstrated. It acts peripherally by blocking impulse generation within the bradykinin sensitive chemoreceptors.

Much investigation has focussed on paracetamol's inhibition of the COX enzyme because its analgesic and antipyretic effects are similar to those of aspirin, the archetype non-steroidal anti-inflammatory drug (NSAID). However, Paracetamol does not have significant anti-inflammatory activity nor does it inhibit production of the pro-clotting Thromboxane A2 (TXAs). In a recent study, Lee and colleagues showed that Paracetamol selectively suppressed peripheral prostaglandin (PG) E2 release and increase COX-2 gene expression in a clinical model of acute inflammation [23].

In our study, the overall incidence of pain during i.v. injection of propofol was $85 \%$ in the control group, $21.7 \%$ in the lidocaïne group and $36.6 \%$ in the paracetamol group ( $\mathrm{p}<0.05)$.

In a recent study, Canbay, et al. have found that i.v. Paracetamol (50 mg) was effective, although not as much 
as lidocaine in decreasing the incidence of pain during i.v. injection of propofol [24]. The authors have shown that its incidence is about 64\% in saline group, $22 \%$ in paracetamol group and $8 \%$ in lidocaïne group [25].

In another study by Ozkan, et al., they found that i.v. pretreatment with Paracetamol $100 \mathrm{mg}$ with tourniquet was as effective as lidocaine, in decreasing the incidence of pain during i.v. injection of propofol. Furthermore, they observed that pracetamol pretreatment with tourniquet was more effective than without in preventing pain on injection [25].

In a prospective, randomized double-blinded study of 250 patients scheduled for elective surgery using a venous retention technique, Borazan, et al. compared the efficacy of the peripheral analgesic action of different doses of paracetamol $\left(0.5 \mathrm{mg} \cdot \mathrm{kg}^{-1}, 1 \mathrm{mg} \cdot \mathrm{kg}^{-1}, 2 \mathrm{mg} \cdot \mathrm{kg}^{-1}\right)$ in reducing the incidence and severity of pain associated with propofol injection and how paracetamol compared with lidocaine $\left(0.5 \mathrm{mg} \cdot \mathrm{kg}^{-1}\right)$. They concluded that pretreatement with paracetamol $2 \mathrm{mg} \cdot \mathrm{kg}^{-1}$, paracetamol $1 \mathrm{mg} \cdot \mathrm{kg}^{-1}$, paracetamol $0.5 \mathrm{mg} \cdot \mathrm{kg}^{-1}$ and lidocaine 0.5 $\mathrm{mg} \cdot \mathrm{kg}^{-1}$ attenuated propofol injection pain when compared with a control group. Paracetamol $1 \mathrm{mg} \cdot \mathrm{kg}^{-1}$ and lidocaine $0.5 \mathrm{mg} \cdot \mathrm{kg}^{-1}$ were equally effective, whereas pretreatment with Paracetamol $2 \mathrm{mg} \cdot \mathrm{kg}^{-1}$ was the most effective treatment studied in reducing propofol injection pain [9].

In our study, we observed that a pretreatment with Paracetamol $100 \mathrm{mg}$ and lidocaine $40 \mathrm{mg}$ with a tourniquet during 120 sec. significantly reduced pain on injection of propofol when compared with a placebo group ( $<$ 0.01). However, we did not find any significant difference between $100 \mathrm{mg}$ Paracetamol and $40 \mathrm{mg}$ lidocaine $(p>0.05)$ in the severity of propofol induced pain.

There are limitations to our study design that may have affected our findings. Firstly, we used a single-fixed dose of paracetamol $(100 \mathrm{mg})$. A greater dose related to patient weight may enhance propofol-induced-pain. Secondly, our study was underpowered to detect any difference in the incidence of moderate to severe pain between lidocaine and paracetamol groups. Finally, a limitation of all studies evaluating vascular pain following propofol administration is the use of a subhypnotic dose of propofol so that reliable pain assessments can be confusing.

\section{Conclusion}

In conclusion, although it may not be much convenient in our daily anaesthesiology practice, we may believe that pretreatment with i.v. paracetamol given with a tourniquet is effective to reduce pain induced by propofol injection and could be an efficient alternative to lidocaine.

\section{Acknowledgements}

This article was sponsored by the research unit UR12SP32.

\section{References}

[1] Abdo El Hamd, M. (2013) The Clinical Pharmacology of Propofol: A Brief Review. Open Journal of Anesthesiology, 3, 367-373. http://dx.doi.org/10.4236/ojanes.2013.38078

[2] Tan, C.H. and Onsiong, M.K. (1998) Pain on Injection of Propofol. Anaesthesia, 53, 468-476. http://dx.doi.org/10.1046/j.1365-2044.1998.00405.x

[3] Kim, K., Kim, Y.S., Lee, D.K., Lim, B.G., Kim, H.Z., Kong, M.H., Kim, N.S. and Lee, I.O. (2013) Reducing the Pain of Microemulsion Propofol Injections: A Double-Blind, Randomized Study of Three Methods of Tourniquet and Lidocaine. Clinical Therapeutics, 35, 1734-1743. http://dx.doi.org/10.1016/j.clinthera.2013.09.018

[4] Jalota, L., Kalira, V., George, V.E., Shi, Y.Y., Hornuss, C., Radke, O., Pace, N.L. and Apfel, C. (2011) Prevention of Pain on Injection of Propofol: Systematic Review and Meta-Analysis. British Medical Journal, 342, d1110. http://dx.doi.org/10.1136/bmj.d1110

[5] Lee, S.K. (2010) Pain on Injection with Propofol. Korean Journal of Anesthesiology, 59, 297-298. http://dx.doi.org/10.4097/kjae.2010.59.5.297

[6] Ahmad, S., De Oliveira Jr., G.S., Fitzgarald, P.C. and McCarthy, R.J. (2013) The Effect of Intravenous Dexamethasone and Lidocaine on Propofol-Induced Vascular Pain: A Randomized Double-Blinded Placebo-Controlled Trial. Pain Research and Treatment, 2013, Article ID: 734531.

[7] Madenoglu, H., Yildiz, K., Dogru, K. and Boyaci, A. (2003) Efficacy of Different Doses of Lidocaine in the Prevention of Pain Due to Propofol Injection: A Randomized, Open-Label Trial in 120 Patients. Current Therapeutic Research, 64, 310-316. http://dx.doi.org/10.1016/S0011-393X(03)00066-3 
[8] Kaya, S., Turhanoglu, S., Karaman, H., Ozgiin, S. and Basak, N. (2008) Lidocaine for Prevention of Propofol Injection-Induced Pain: A Prospective, Randomized, Double-Blind, Controlled Study of the Effect of Duration of Venous Occlusion with a Tourniquet in Adults. Current Therapeutic Research, 69, 29-35. http://dx.doi.org/10.1016/j.curtheres.2008.02.005

[9] Borazan, H., Erdem, T.B., Kececioglu, M. and Otelcioglu, S. (2010) Prevention of Pain on Injection of Propofol: A Comparison of Lidocaine with Different Doses of Paracetamol. European Journal of Anaesthesiology, 27, $253-257$. http://dx.doi.org/10.1097/EJA.0b013e328330eca2

[10] Anderson, B.J. (2008) Paracetamol (Acetaminophen): Mechanisms of Action. Pediatric Anesthesia, 18, 915-921. http://dx.doi.org/10.1111/j.1460-9592.2008.02764.x

[11] Macario, A., Weinger, M., Carney, S. and Kim, A. (1999) Which Clinical Anesthesia Outcomes Are Important to Avoid? The Perspective of Patients. Anesthesia and Analgesia, 89, 652-658.

[12] Sim, J.Y., Lee, S.H., Park, D.Y., Jung, J.A., Ki, K.H., Lee, D.H. and Noh, G.J. (2009) Pain on Injection with Microemulsion Propofol. British Journal of Clinical Pharmacology, 67, 316-325. http://dx.doi.org/10.1111/j.1365-2125.2008.03358.x

[13] Ando, R. and Watanabe, C. (2005) Characteristics of Propofol-Evoked Vascular Pain in Anaesthetized Rats. British Journal of Anaesthesia, 95, 384-392. http://dx.doi.org/10.1093/bja/aei184

[14] Wallentine, C.B., Shimode, N., Egan, T.D. and Pace, N.L. (2011) Propofol in a Modified Cyclodextrin Formulation: First Human Study of Dose-Response with Emphasis on Injection Pain. Anesthesia and Analgesia, 113, 738-741.

[15] Yamakage, M., Iwasaki, S., Satoh, J.I. and Namiki, A. (2005) Changes in Concentrations of Free Propofol by Modification of the Solution. Anesthesia and Analgesia, 101, 385-388. http://dx.doi.org/10.1213/01.ANE.0000154191.86608.AC

[16] Baker, M.T. and Naguib, M. (2005) Propofol: The Challenges of Formulation. Anesthesiology, 103, 860-876. http://dx.doi.org/10.1097/00000542-200510000-00026

[17] Kam, E., Abdul-Latif, M.S. and McCluskey, A. (2004) Comparison of Propofol-Lipuro with Propofol Mixed with Lidocaine $10 \mathrm{mg}$ on Propofol Injection Pain. Anaesthesia, 59, 1167-1169. http://dx.doi.org/10.1111/j.1365-2044.2004.03964.x

[18] Le Guen, M., Grassin-Delyle, S., Cornet, C., Genty, A., Chazot, T., Dardelle, D., Liu, N., Dreyfus, J.F., Mazoit, J.X., Devillier, P., Alvarez, J.C., Sessler, D.I. and Fischler, M. (2014) Comparison of the Potency of Different Propofol Formulations: A Randomized, Double-Blind Trial Using Closed-Loop Administration. Anesthesiology, 120, 355-364.

[19] Tarmiz, K., Ferhi, F., Toumi, M., Slama, A. and BenJazia, K. (2009) Efficacicité d’une faible dose de kétamine dans la réduction de la douleur liée à l’injection de propofol. Annales Française Anesthésie Réanimation, 28, 177-178. http://dx.doi.org/10.1016/j.annfar.2008.12.014

[20] Kim, H.S., Cho, K.R., Lee, J.H., Kim, Y.H., Lim, S.H., Lee, K.M., Cheong, S.H., Kim, Y.J., Shin, C.M. and Lee, J.Y. (2010) Prevention of Pain During Injection of Microemulsion Propofol: A Lication of Lidocaine Mixture and the Optimal Dose of Lidocaine. Korean Journal of Anesthesiology, 59, 310-313. http://dx.doi.org/10.4097/kjae.2010.59.5.310

[21] Sasaki, T., Okamura, S. and Kisara, A. (1999) Effect of Lidocaine on Pain Caused by Injection of Propofol: Comparison of Three Methods at Two Injection Rates. Journal of Anesthesia, 13, 14-16. http://dx.doi.org/10.1007/s005400050015

[22] Picard, P. and Tramèr, M.R. (2000) Prevention of Pain on Injection with Propofol: A Quantitative Systematic Review. Anesthesia \& Analgesia, 90, 963-969. http://dx.doi.org/10.1213/00000539-200004000-00035

[23] Lee, Y.S., Kim, H., Brahim, J.S., Rowan, J., Lee, G. and Dionne, R.A. (2007) Acetaminophen Selectively Suppresses Peripheral Prostaglandin E2 Release and Increases COX-2 Gene Expression in a Clinical Model of Acute Inflammation. Pain, 129, 279-286. http://dx.doi.org/10.1016/j.pain.2006.10.020

[24] Canbay, O., Celebi, N., Arun, O., Karagöz, A.H., Sarıcaoglu, F. and Özgen, S. (2008) Efficacy of Intravenous Acetaminophen and Lidocaine on Propofol Injection Pain. British Journal of Anaesthesia, 100, 95-98. http://dx.doi.org/10.1093/bja/aem301

[25] Ozkan, S., Sen, H., Sizlan, A., Yanarates, O., Mutlu, M. and Dagli, G. (2011) Comparison of Acetaminophen (with or without Tourniquet) and Lidocaine in Propofol Injection Pain. Bulletin of Clinical Psychopharmacology, 21, 100-104. http://dx.doi.org/10.5455/bcp.20110408022041 